\title{
SIRT6-specific inhibitor OSS-128167 exacerbates diabetic cardiomyopathy by aggravating inflammation and oxidative stress
}

\author{
YIBO HUANG ${ }^{1}$, JUNKAI ZHANG ${ }^{2}$, DONGDONG XU ${ }^{3}$, YU PENG ${ }^{2}$, YUAN JIN $^{2}$ and LEI ZHANG ${ }^{2}$ \\ Departments of ${ }^{1}$ Anesthesiology, ${ }^{2}$ Pain Medicine and ${ }^{3}$ Neurology, The First Affiliated Hospital, \\ Wenzhou Medical University, Wenzhou, Zhejiang 325035, P.R. China
}

Received April 6, 2020; Accepted December 8, 2020

DOI: $10.3892 / \mathrm{mmr} .2021 .12006$

\begin{abstract}
Diabetic cardiomyopathy (DCM) is a serious complication of diabetes, which importantly contributes to the increased mortality of patients with diabetes. The development of DCM is accompanied by numerous pathological mechanisms, including oxidative stress and chronic inflammation. Accordingly, the present study aimed to determine the effects of the sirtuin 6 (SIRT6) inhibitor OSS-128167 on DCM using a mouse model of streptozotocin (STZ)-induced diabetes and high glucose (HG)-treated cardiomyocytes. C57BL/6 mice were intraperitoneally injected with STZ for 5 days to simulate the diabetic cardiomyopathy model. Mice with STZ-induced diabetes (STZ-DM1) were orally administered OSS-128167 (20 or $50 \mathrm{mg} / \mathrm{kg}$ ) through gavage every other day. The expression of SIRT6 in myocardial tissue was detected using western blotting. Tissue staining (hematoxylin and eosin and Masson's trichrome) was used to characterize myocardial structure, TUNEL fluorescent staining was used to detect myocardial apoptosis, and immunohistochemical staining was used to detect the expression of inflammatory factors in myocardial tissue. Dihydroethidium staining and a malondialdehyde (MDA) detection kit were used to detect the oxidative stress levels in myocardial tissues. In vitro, $\mathrm{H} 9 \mathrm{c} 2$ cells were pre-incubated with OSS-128167 for $1 \mathrm{~h}$ and then stimulated with HG (33 mM) for various durations. Expression levels of fibrosis markers, collagen-1 and transforming
\end{abstract}

Correspondence to: Dr Lei Zhang, Department of Pain Medicine, The First Affiliated Hospital, Wenzhou Medical University, 2 Fuxue Street, Wenzhou, Zhejiang 325035, P.R. China

E-mail: sto_zhanglei@163.com

Abbreviations: DCM, diabetic cardiomyopathy; SIRT6, sirtuin 6; STZ, streptozotocin; ROS, reactive oxygen species; TNF- $\alpha$, tumor necrosis factor- $\alpha$; DHE, dihydroethidium; MDA, malondialdehyde; HG, high glucose; COL, collagen; TGF- $\beta$, transforming growth factor $\beta$; CTGF, connective tissue growth factor; Bcl-2, B-cell lymphoma-2; Bax, Bcl-2-associated X; cle-PARP, cleaved poly ADP-ribose polymerase; 3-NT, 3-nitrotyrosine

Key words: DCM, SIRT6, OSS-128167, inflammation, oxidative stress growth factor (TGF)- $\beta$, apoptosis-related proteins, Bax, Bcl-2 and cleaved-poly ADP-ribose polymerase, tumor necrosis factor- $\alpha$ and the oxidative stress metabolite, 3-nitrotyrosine were analyzed using western blotting and reverse transcription-quantitative PCR. Commercially available kits were used to detect the activity of caspase- 3 and the content of MDA in the $\mathrm{H} 9 \mathrm{c} 2$ cell line. The corresponding results demonstrated that OSS-128167 aggravated diabetes-induced cardiomyocyte apoptosis and fibrosis in mice. Mechanistically, OSS-128167 was revealed to increase the levels of inflammatory factors and reactive oxygen species (ROS) in vitro and in vivo. In conclusion, OSS-128167 facilitated the inflammatory response and promoted the production of ROS while aggravating DCM development. These findings indicated that SIRT6 may target two closely combined and interacting pathological processes, the inflammatory response and oxidative stress, and may serve as a potentially advantageous therapeutic target.

\section{Introduction}

Diabetic cardiomyopathy (DCM) is a complication associated with serious microangiopathy, which is responsible for the increased risk of morbidity and mortality in patients suffering from diabetes (1). It has been estimated that DCM will affect -552 million patients globally by 2030 , according to an official report $(2,3)$. Cardiac dysfunction and pathological structural changes, including cardiac hypertrophy, cardiac fibrosis, cardiomyocyte apoptosis, mitochondrial dysfunction and autophagy, occur in the development of DCM (4-6). As previously reported, several pathophysiological mechanisms may affect the pathogenesis of DCM, including unbalanced energy metabolism, inflammation and oxidative stress (7-9). Elucidating the pathogenesis of DCM requires urgent investigation to provide an effective treatment strategy. Inflammation and oxidative stress have been reported to markedly affect the development and progression of diabetes, as well as its complications $(8,9)$. Additionally, previous studies have indicated that uncontrolled inflammation and oxidative stress may contribute to cardiac dysfunction in diabetic cardiac tissues $(10,11)$. Extensive research has also revealed that mitochondrial injury may serve as a central mediator in the pathology of DCM $(12,13)$. Therefore, identifying potential therapeutic strategies that target chronic inflammation and oxidative stress may aid in the management of DCM. 
Sirtuins (SIRTs) are a highly conserved family of $\mathrm{NAD}^{+}$-dependent deacetylases, which may have a broad impact on numerous biological pathways (14). Among the SIRTs, SIRT6 is a nuclear protein known to deacetylate histone H3 lysine 9 and $\mathrm{H} 3$ lysine $56(15,16)$. SIRT6 has been reported to serve a crucial role in human telomere and genome stabilization (17), glucose and lipid metabolism (18), and inflammation and oxidative stress $(19,20)$. In this regard, the SIRT6 may be potentially used to treat diabetes, immune-mediated disorders and cardiovascular diseases. Previous studies have demonstrated that knockout of SIRT6 can aggravate macrophage foaming and exacerbate atherosclerosis, whereas increased expression of SIRT6 can alleviate endothelial cell dysfunction $(21,22)$. According to these previous studies, SIRT6 may have a favorable role in cardiovascular disease. However, the role and mechanism of SIRT6 in DCM has been seldom reported.

The present study aimed to verify whether the SIRT6 inhibitor OSS-128167 aggravated myocardial injury in DCM. A mouse model of streptozotocin (STZ)-induced type 1 diabetes and cultured cardiomyocytes were employed in the present study. The corresponding results supported the deleterious effect of OSS-128167 on DCM via the aggravation of inflammation and oxidative stress. By analyzing experimental results in vivo and in vitro, it was confirmed that SIRT6 may be considered a potential therapeutic target in treating DCM.

\section{Materials and methods}

Cell culture. The H9c2 cell line consists of subclonal cells obtained from the heart tissue of embryonic rats. H9c2 cells were provided by the Shanghai Institute of Biochemistry and Cell Biology, Chinese Academy of Sciences. H9c2 cells were cultured in DMEM (Gibco; Thermo Fisher Scientific, Inc.) supplemented with $5.5 \mathrm{mM}$ D-glucose, $100 \mathrm{U} / \mathrm{ml}$ penicillin, 10\% FBS (Gibco; Thermo Fisher Scientific, Inc.) and $100 \mathrm{mg} / \mathrm{ml}$ streptomycin at $37^{\circ} \mathrm{C}$ in a humidified $5 \% \mathrm{CO}_{2}$ incubator. Prior to experimentation, $\mathrm{H} 9 \mathrm{c} 2$ cells were pretreated with 20 and $50 \mu \mathrm{M}$ OSS-128167 (Selleck Chemicals) for $1 \mathrm{~h}$, followed by stimulation with high glucose $(\mathrm{HG} ; 33 \mathrm{mM})$ for various durations (both steps were conducted at $37^{\circ} \mathrm{C}$ ). For analysis of transforming growth factor (TGF)- $\beta$, collagen (COL)-1, Bax, Bcl-2 and cleaved-poly ADP-ribose polymerase (PARP) protein, cells were stimulated with $\mathrm{HG}$ for $36 \mathrm{~h}$; for RT-qPCR analysis of TGF- $\beta$, COL-1, Bax, Bcl-2 and cleaved-PARP mRNA, cells were stimulated with HG for $12 \mathrm{~h}$; for western blotting of tumor necrosis factor (TNF)- $\alpha$ and 3-nitrotyrosine (NT), cells were stimulated with HG for $24 \mathrm{~h}$; for RT-qPCR analysis of TNF- $\alpha$, cells were stimulated with $\mathrm{HG}$ for $8 \mathrm{~h}$; and to detect malondialdehyde (MDA) levels, cells were stimulated with $\mathrm{HG}$ for $4 \mathrm{~h}$.

Reagents. Selective SIRT6 antagonist OSS-128167 was dissolved in dimethyl sulfoxide (DMSO) to obtain $50 \mathrm{mM}$ stock solution, which was diluted in DMSO for use in further experiments. The chemical structure of OSS_128167 is shown in Fig. 1C. DMSO had a final concentration of $0.1 \%(\mathrm{v} / \mathrm{v})$. All other chemical biological agents were purchased from Sigma-Aldrich; Merck KGaA. Antibodies for SIRT6, TNF- $\alpha$,
COL-1, TGF- $\beta$, PARP, 3-NT and GAPDH were purchased from Abcam.

Animal experiments. Animals were provided by the Animal Center of Wenzhou Medical University. The present study was approved by the Wenzhou Medical University Animal Policy and Welfare Committee (Wenzhou, China), and experiments were conducted according to the National Institutes of Health (NIH) guidelines regarding the care and use of laboratory animals. A total of 40 male C57BL/6 mice (weight, 22-26 g; age, 10 weeks; Riken BioResource Center of Japan) were housed in an environment with room temperature maintained at $22 \pm 2.0^{\circ} \mathrm{C}$ and humidity at $50 \pm 5 \%$. In addition, they were kept under a 12-h light/dark cycle, and were fed with a standard rodent diet and water. After 1 week of acclimation, mice were intraperitoneally injected with $50 \mathrm{mg} / \mathrm{kg} \mathrm{STZ}$ (Sigma-Aldrich; Merck KGaA) formulated in citrate buffer (100 mM, pH 4.5) for 5 consecutive days. A glucometer was used to detect the levels of fasting blood-glucose (FBG). OSS-128167 intervention was commenced once it was confirmed that type 1 diabetes mellitus (FBG $>12 \mathrm{mmol} / \mathrm{l}$ ) was successfully established. Mice with STZ-induced diabetes (STZ-DM1) were orally administered OSS-128167 (20 or $50 \mathrm{mg} / \mathrm{kg}$ ) or vehicle (1\% CMC-Na) through gavage every other day ( $\mathrm{n}=10 /$ group). As shown in Fig. S1A, the body weight and blood glucose were recorded at certain time points. A total of 16 weeks after administration, the mice were anesthetized with ketamine hydrochloride $(100 \mathrm{mg} / \mathrm{kg}$ body weight; Ketanest; Pfizer) and xylazine hydrochloride $(16 \mathrm{mg} / \mathrm{kg}$ body weight; Rompun 2\%; Bayer). Blood was collected from the retro-orbital vein after the mice were anesthetized; $500 \mu \mathrm{l}-1 \mathrm{ml}$ blood was collected. Subsequently, the mice were euthanized by cervical dislocation, the thoracic cavity was opened, and the heart was removed after perfusion with normal saline. The heart tissues were embedded in paraffin, and maintained at $65^{\circ} \mathrm{C}$ for $1.5 \mathrm{~h}$. Paraffin samples were subsequently stored at $4^{\circ} \mathrm{C}$, while other heart tissues were placed in liquid nitrogen for snap-freezing before further analysis. The collected blood samples were centrifuged for $5 \mathrm{~min}\left(1,500 \mathrm{x} \mathrm{g}, 4^{\circ} \mathrm{C}\right)$ to collect the serum.

Immunohistochemistry. Fixed heart sections $(5-\mu \mathrm{m})$ described above were incubated with $3 \% \mathrm{H}_{2} \mathrm{O}_{2}$ for 30 min and blocked for 30 min with $2 \%$ bovine serum albumin (Sigma-Aldrich; Merck $\mathrm{KGaA}$ ), both at room temperature in PBS. Slides were then incubated overnight with a primary antibody against TNF- $\alpha$ (1:500; Santa Cruz Biotechnology, Inc.; cat. no. sc-52746) at $4^{\circ} \mathrm{C}$, followed by incubation with a secondary antibody (1:200; Santa Cruz Biotechnology, Inc.; cat. no. sc-516102) for $1 \mathrm{~h}$ and with DAB (A:B, 1:20) for $5 \mathrm{~min}$ at room temperature. Hematoxylin was used to stain the cell nuclei for $3 \mathrm{~min}$ at room temperature and resin was used to seal the dehydrated sections. A light microscope (magnification, $\mathrm{x} 400$; Nikon Corporation) was used to capture the images.

TUNEL staining. TUNEL staining was performed using the TUNEL Apoptosis Detection kit (R\&D Systems, Inc.), according to the manufacturer's protocol. Heart tissue sections were counterstained with DAPI (Beyotime Institute of Biotechnology) at room temperature for $5 \mathrm{~min}$. Images were 
captured using the Leica A1 laser confocal microscope (Leica Microsystems $\mathrm{GmbH}$ ). The number of TUNEL-positive cells was counted using Image J 1.52a software (NIH) in three randomly selected fields per sample.

Hematoxylin and eosin $(H \& E)$ staining for morphology and Masson's trichrome staining for fibrosis. Heart tissues were fixed in $4 \%$ paraformaldehyde at room temperature for $24 \mathrm{~h}$, embedded in paraffin and sectioned into 5- $\mu \mathrm{m}$ thick samples. The tissue sections were incubated at $60-70^{\circ} \mathrm{C}$ for $4-6 \mathrm{~h}$, then deparaffinized in xylene at room temperature for $10 \mathrm{~min}$ and another subsequent $15 \mathrm{~min}$ to complete deparaffinization. The sections were then rehydrated in a descending series of ethanol, prior to being immersed in PBS trice at room temperature for $5 \mathrm{~min}$ each and finally washed in distilled water at room temperature for $20 \mathrm{~min}$ for subsequent staining. $\mathrm{H} \& \mathrm{E}$ and Masson's trichrome staining were used to evaluate the intima-media thickness and fibrosis content of the tissues.

For H\&E staining (Beijing Solarbio Science \& Technology Co., Ltd.; cat. no. G1120), the sections were stained with eosin for $2 \mathrm{~min}$, wash in distilled water for $20 \mathrm{~min}$ and stained with hematoxylin for $5 \mathrm{~min}$ (all at room temperature). Subsequently, sections were washed in distilled water for 5 min thrice, dehydrated using an ascending ethanol series and the slides were then mounted with neutral gum.

For Masson's trichrome staining (Beijing Solarbio Science \& Technology Co., Ltd.; cat. no. G1340), the sections were stained with ponceau staining solution dye for $5 \mathrm{~min}$, washed with distilled water for 1 min twice and incubated with $1 \%$ phosphotungstic acid solution for $5 \mathrm{~min}$. Then, the sections were incubated with aniline blue dye solution for $5 \mathrm{~min}$, treated with $1 \%$ glacial acetic acid water for $1 \mathrm{~min}$, dehydrated using an ascending ethanol series and then stored at room temperature. The entire staining process was performed at room temperature. A light microscope (magnification, $\mathrm{x} 400$; Nikon Corporation) was used to observe the stained sections.

Determination of superoxide production and the levels of cellular $\mathrm{H}_{2} \mathrm{O}_{2}$. Dihydroethidium (DHE) staining was used to evaluate superoxide production. Briefly, the isolated mouse heart tissues were embedded in OCT compound (Sakura Finetek USA, Inc.) immediately after the heart tissues were excised from the mice, after which they were split into frozen $5-\mu \mathrm{m}$ sections. The sections were then incubated in DHE in PBS (10 mmol/l) for $45 \mathrm{~min}$ in a dark and moist container at $37^{\circ} \mathrm{C}$. DHE was oxidized upon the reaction with superoxide to ethidium bromide, which binds to DNA in the nucleus and fluoresces red (23). DHE dilutions were prepared as 1:5,000 to treat the sections, and images were captured using the Leica A1 laser confocal microscope (Leica Microsystems GmbH).

Determination of MDA. An MDA kit (Beyotime Institute of Biotechnology; cat. no. S0131S) was used to determine the levels of MDA in cells and tissues, according to the manufacturer's protocol.

Caspase 3 activity assay. PBS and 0.025\% trypsin (T6325-25 g; Macklin Inc.) were used to wash and digest H9c2 cells, respectively. The cells then underwent centrifugation at $600 \mathrm{xg}$ for $5 \mathrm{~min}$ at $4^{\circ} \mathrm{C}$. The supernatant was discarded, and PBS was used to wash the precipitate twice. Lysis buffer was then added at a ratio of $100 \mu \mathrm{l}$ lysate $/ 2$ million cells according to the kit's instructions (cat. no. C1115; Beyotime Institute of Biotechnology). The mixture then underwent centrifugation at $20,000 \mathrm{x}$ g for $15 \mathrm{~min}$ at $4^{\circ} \mathrm{C}$, after $15 \mathrm{~min}$ of lyophilization in an ice bath. Centrifuged supernatant $(50 \mu \mathrm{l})$ and Ac-DEVD-pNA $(10 \mu 1)$ were mixed in a 96-well plate (without generating air bubbles while mixing). Finally, the 96-well plate was incubated for $90 \mathrm{~min}$ at $37^{\circ} \mathrm{C}$, after which a spectrophotometer was used to measure the optical density value at an absorbance of $405 \mathrm{~nm}$.

Reverse transcription quantitative ( $R T-q P C R)$. According to a standard protocol, $\mathrm{H} 9 \mathrm{c} 2$ cells were homogenized in TRIzol $^{\circledR}$ (Invitrogen; Thermo Fisher Scientific, Inc.) to extract RNA. The two-step M-MLV Platinum SYBR Green qPCR SuperMix- UDG kit (Thermo Fisher Scientific, Inc.) and Eppendorf Mastercycler eprealplex detection system (Eppendorf) were used to conduct RT and qPCR. Reverse transcription was performed at $37^{\circ} \mathrm{C}$ for $15 \mathrm{~min}$ and $85^{\circ} \mathrm{C}$ for $5 \mathrm{sec}$, and then maintained at $4^{\circ} \mathrm{C}$. The following thermocycling conditions were used for the qPCR: Initial denaturation at $95^{\circ} \mathrm{C}$ for $3 \mathrm{~min}$; followed by 50 cycles at $95^{\circ} \mathrm{C}$ for $15 \mathrm{sec}$ and $60^{\circ} \mathrm{C}$ for $30 \mathrm{sec}$, and a melting curve stage at $95^{\circ} \mathrm{C}$ for $15 \mathrm{sec}$ and $60^{\circ} \mathrm{C}$ for $1 \mathrm{~min}$. The primers were provided by Thermo Fisher Scientific, Inc. and primer sequences are presented in Table SI. The Tm values were normalized to $\beta$-actin. The $2^{-\Delta \Delta C q}$ method was used to quantify the expression levels (24).

Immunoblotting. Cells were collected and underwent lysis using a buffer (cat. no. AR0103-100; Boster Biological Technology). Protein was quantified using a BCA assay and protein lysates $(40 \mu \mathrm{g})$ were then separated by $10 \%$ SDS-PAGE and electrotransferred to polyvinylidene fluoride membranes. The membranes then underwent $1 \mathrm{~h}$ of blocking in TBS ( $\mathrm{pH}$ 7.6) containing 5\% non-fat milk and $0.05 \%$ Tween 20 at room temperature. The membranes were incubated with primary antibodies overnight at $4{ }^{\circ} \mathrm{C}$ and with the secondary antibodies at room temperature for $2 \mathrm{~h}$. TGF- $\beta$ (1:500; cat. no. sc-146), B-cell lymphoma-2 (Bcl-2; 1:500; cat. no. sc-7382), NF- $\kappa B$ P65 (1:500; cat. no. sc-7151), cleaved (cle)-PARP (1:500; cat. no. sc-56196) and Bcl-2-associated X (Bax; 1:500; cat. no. sc-7480) antibodies were purchased from Santa Cruz Biotechnology, Inc. SIRT6 (1:1,000; cat. no. ab191385), COL-1 (1:1,000; cat. no. ab34710), TNF- $\alpha$ (1:1,000; cat. no. ab6671), 3 -NT (1:1,000; cat. no. ab61392) and GAPDH (1:1,000; cat. no. ab8245) antibodies were provided by Abcam. The primary and secondary antibodies (1:1,000; Cell Signaling Technology, Inc.; cat. nos. 7076 and 7074) were diluted in TBS- $0.05 \%$ Tween-20 prior to use. The enhanced chemiluminescence kit (Bio-Rad Laboratories, Inc.) was used to detect the specific bands. Densitometric analysis was performed using Image Lab 5.1 software (Bio-Rad Laboratories, Inc.).

Statistical analysis. Experiments were repeated three times and data were expressed as the mean \pm SEM. GraphPad Pro Prism 6.0 (GraphPad Software, Inc.) was used to conduct statistical analyses. Student's t-test was used to analyze the differences between two datasets, while a one-way ANOVA 

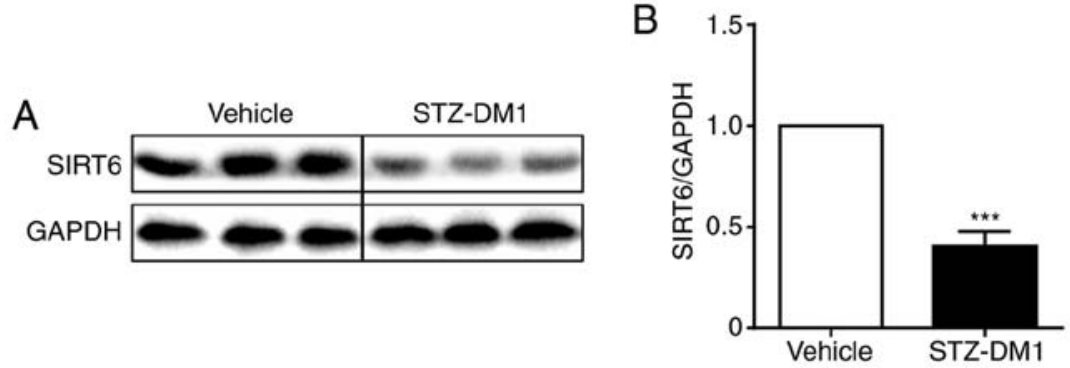

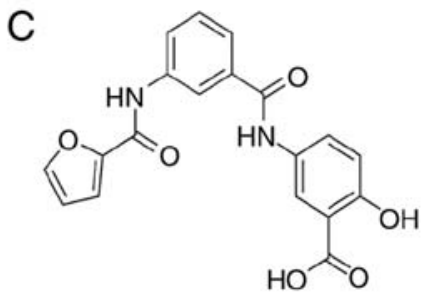

OSS_128167

D
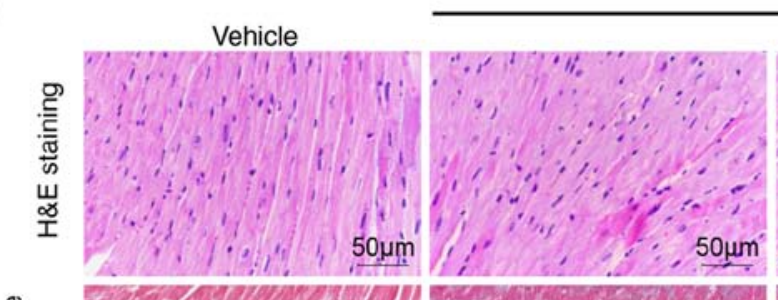

STZ-DM1
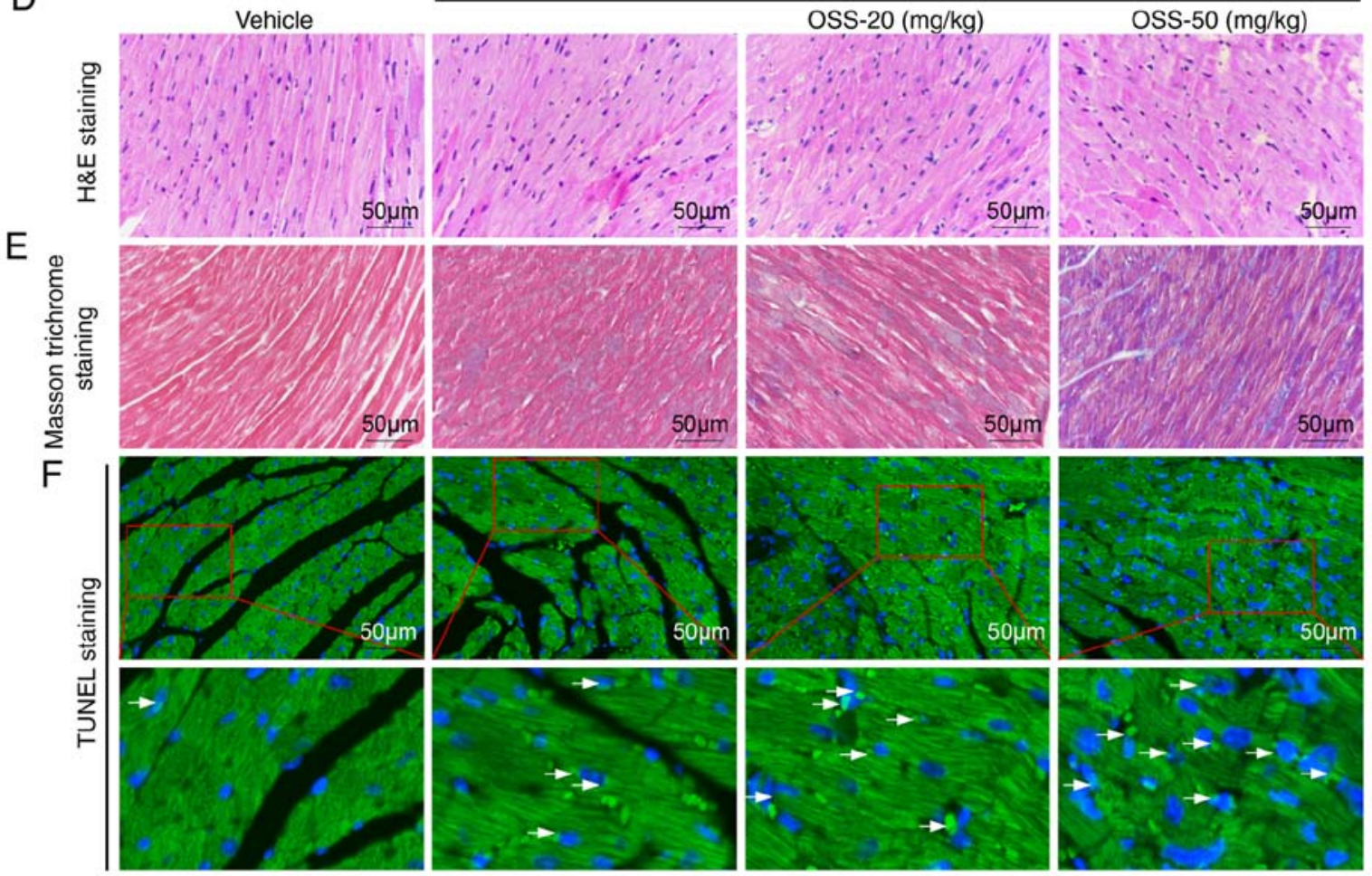

Figure 1. OSS-128167 aggravates hyperglycemia-induced cardiac damage. (A and B) Representative images of immunoblot analysis of SIRT6 in cardiac tissue. GAPDH was used as a loading control. (C) Chemical structure of the SIRT6 inhibitor OSS-128167. (D) Representative histomorphometric images of H\&E staining of myocardial tissues from each group fixed with formalin (magnification, x400). (E) Representative micrographs of Masson trichrome staining of myocardial tissues (magnification, $\mathrm{x} 400$ ). (F) Heart tissues underwent TUNEL assay (magnification, $\mathrm{x} 400$ ); the lower panel is a partially enlarged image of the upper panel (magnification, x1,200). $\mathrm{n}=6-9 .{ }^{* * * *} \mathrm{P}<0.001$ vs. Vehicle. SIRT6, sirtuin 6; H\&E, hematoxylin and eosin; STZ, streptozotocin; STZ-DM1, STZ-induced diabetes.

and Bonferroni correction were used to assess multiple comparisons. $\mathrm{P}<0.05$ was considered to indicate a statistically significant difference.

\section{Results}

SIRT6 inhibitor OSS-128167 aggravates cardiac pathological abnormalities in mice with type 1 diabetes caused by hyperglycemia. Type 1 diabetes in male C57BL/6 mice was induced by successively injecting a low dose of STZ intraperitoneally. In the STZ-mediated mouse model of type 1 diabetic cardiomyopathy (STZ-DM1), the protein expression levels of SIRT6 were significantly lower in myocardial tissues of STZ-DM1 mice compared with those in the non-diabetic controls (Fig. 1A and B). Subsequently, the mouse model of type 1 diabetic cardiomyopathy was employed to determine whether OSS-128167 (Fig. 1C) aggravated diabetes-induced cardiac damage. Diabetic mice were treated with OSS-128167
(20 and $50 \mathrm{mg} / \mathrm{kg}$ ) for 2 months, and the effects of OSS-128167 on diabetic cardiac morphology were examined. As shown using H\&E and Masson's trichrome staining, diabetic hearts exhibited an abnormal structure compared with the hearts of normal mice. Compared with the vehicle group, H\&E staining showed myocardial fibrosis in the STZ-DM1 group was disordered, while Masson's trichrome staining showed increased myocardial fibrosis in the STZ-DM1 group. Notably, these structural abnormalities were aggravated by treatment of diabetic mice with OSS-128167 (Fig. 1D and E). Finally, TUNEL staining was used to detect myocardial tissue apoptosis. As shown in Fig. 1F, OSS-128167 treatment markedly increased cell apoptosis, as indicated by an increase in the TUNEL-positive staining area. Notably, it was revealed that such adverse effects imposed by OSS-128167 on cardiac morphology were not caused by metabolic changes. OSS-128167 treatment had no impact on FBG and serum insulin levels (Fig. S1B and C). Moreover, compared with that 
A
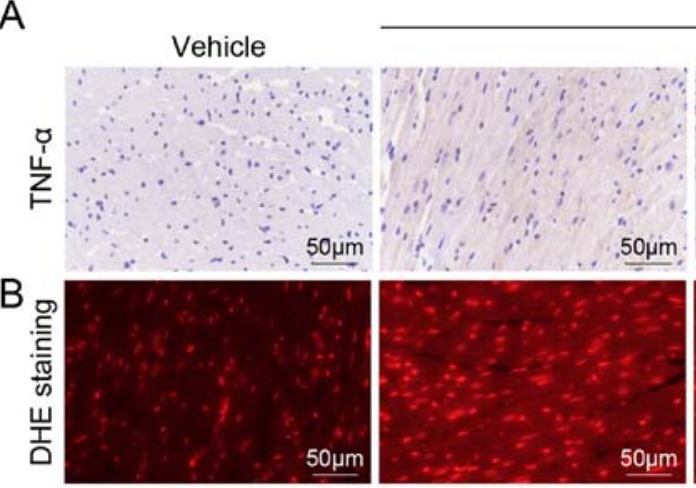

STZ-DM1

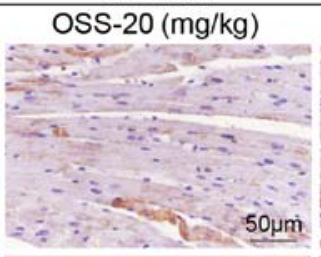

OSS-50 $(\mathrm{mg} / \mathrm{kg})$
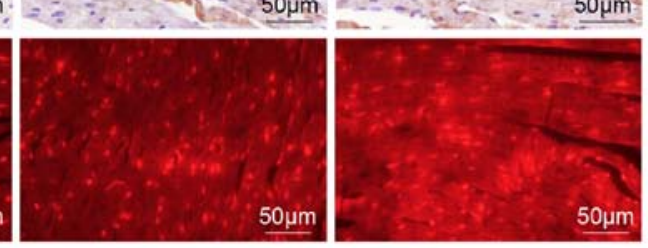

C

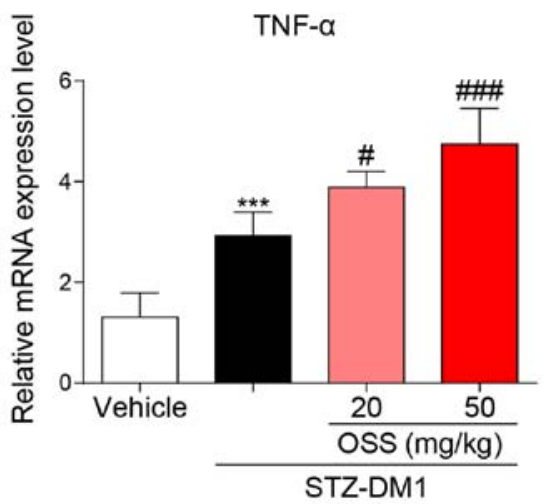

D

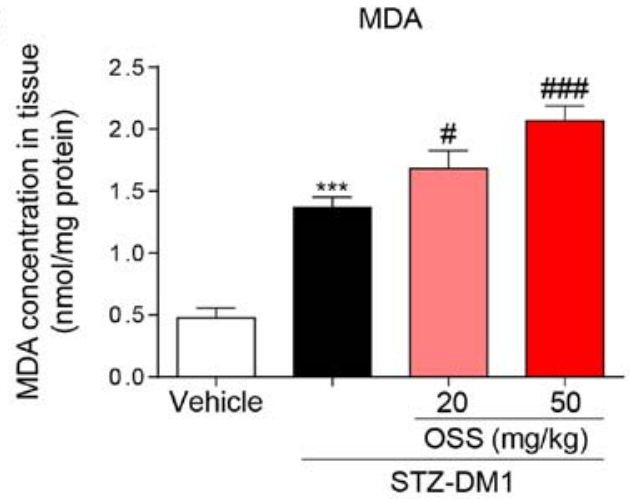

Figure 2. OSS-128167 aggravates myocardial inflammation and oxidative stress. (A) DAB staining (brown) was used to determine TNF- $\alpha$ immunoreactivity in mouse heart tissues (magnification, x400). (B) Reactive oxygen species levels in heart tissues were detected using DHE staining (magnification, x400). (C) Reverse transcription-quantitative PCR was used to determine the mRNA expression levels of TNF- $\alpha$ in myocardial tissues. (D) Levels of MDA in heart tissues. $\mathrm{n}=6-9$. ${ }^{* * *} \mathrm{P}<0.001$ vs. Vehicle; ${ }^{\#} \mathrm{P}<0.05,{ }^{\# \#} \mathrm{P}<0.001$ vs. STZ-DM1. STZ, streptozotocin; STZ-DM1, STZ-induced diabetes; TNF- $\alpha$, tumor necrosis factor- $\alpha$; DHE, dihydroethidium; MDA, malondialdehyde.

in the vehicle group, heart weight/body weight (HW/BW) was significantly increased in the STZ-DM1 and OSS-128167 treatment groups (Fig. S1D). However, no significant difference in HW/BW was apparent in the OSS-128167 treatment group compared with in the STZ-DM1 group. These results indicated that OSS-128167 exacerbated cardiac structural destruction, and myocardial fibrosis and apoptosis in type 1 diabetic mice.

OSS-128167 aggravates diabetes-induced myocardial inflammation and oxidative stress. In order to determine the potential molecular mechanisms underlying the deleterious effect exerted by OSS-128167 in vivo, the expression levels of inflammatory mediators and the levels of oxidative stress in the myocardium were detected in response to OSS-128167. Notably, the mRNA and protein expression levels of the inflammatory factor TNF- $\alpha$ were significantly increased in the STZ-DM1 group compared with those detected in the vehicle group; a further increase in these levels was also detected in the OSS-128167 treatment group (Fig. 2A and C). The biological effects of OSS-128167 on oxidative stress were further detected in the hearts of each group. DHE and MDA kits were used to detect oxidative stress in the myocardium; higher levels of superoxide and MDA were detected in the hearts of mice in the OSS-128167 group compared with those in the STZ-DM1 group (Fig. 2B and D). These results indicated that OSS-128167 aggravated myocardial injury in DCM by exacerbating hyperglycemia-mediated inflammation and oxidative stress.

OSS-128167 aggravates HG-induced cell fibrosis and apoptosis of $\mathrm{H} 9 \mathrm{c} 2 \mathrm{cells}$. The results of the present study were verified by observing cultured cardiomyocytes in vitro and the potential molecular mechanisms were explored. Briefly, $\mathrm{H} 9 \mathrm{c} 2$ cells were cultured and treated with $\mathrm{HG}(33 \mathrm{mM})$ to simulate hyperglycemia. Following treatment, immunoblotting was conducted to assess the expression levels of biomarkers for cell fibrosis (Fig. 3A). OSS-128167 significantly increased the expression levels of pro-fibrotic markers COL- 1 and TGF- $\beta$ compared with in the HG group (Fig. 3A). In addition, OSS-128167 induced a dose-dependent increase in the mRNA expression levels of COL- 1 and TGF- $\beta$, as well as CTGF (Fig. 3B-D). The present in vivo studies revealed that OSS-128167 increased apoptosis in the heart tissues of mice. Consequently, the effects of OSS-128167 on $\mathrm{HG}$-induced apoptosis in $\mathrm{H} 9 \mathrm{c} 2$ cells were examined. Under a HG challenge, the expression levels of the apoptotic biomarker Bax were markedly upregulated by OSS-128167, whereas the opposite was observed for Bcl-2 (Fig. 3E). Treatment with OSS-128167 also significantly reduced the protein expression ratio of Bcl-2/Bax compared with that in the HG group (Fig. 3F). As shown in Fig. 3G, OSS-128167 readily increased the protein expression levels of cle-PARP, which were initially induced by HG. Moreover, caspase-3 
A

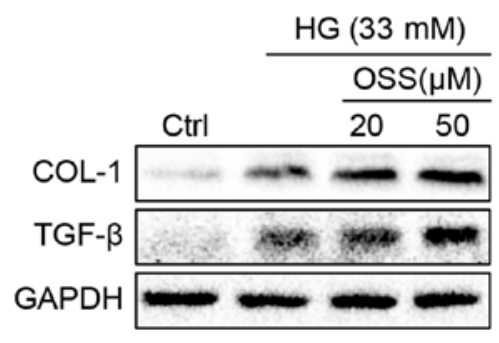

C

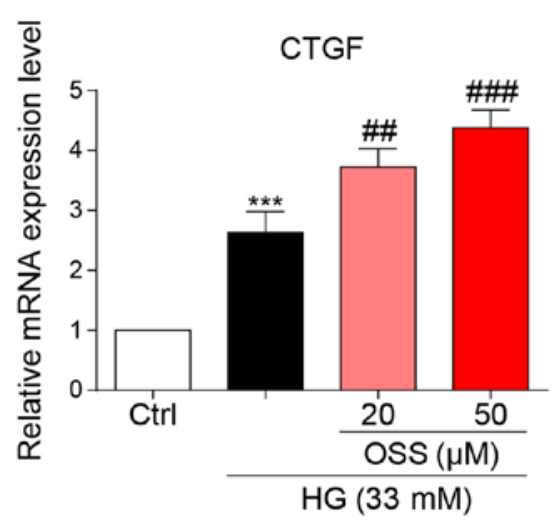

E

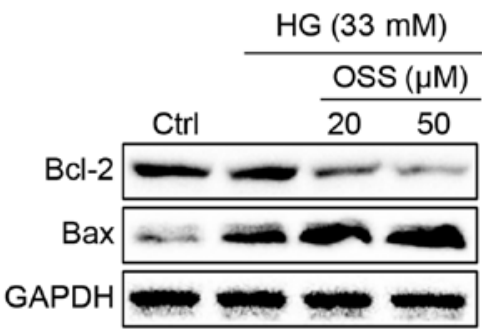

$\mathrm{B}$

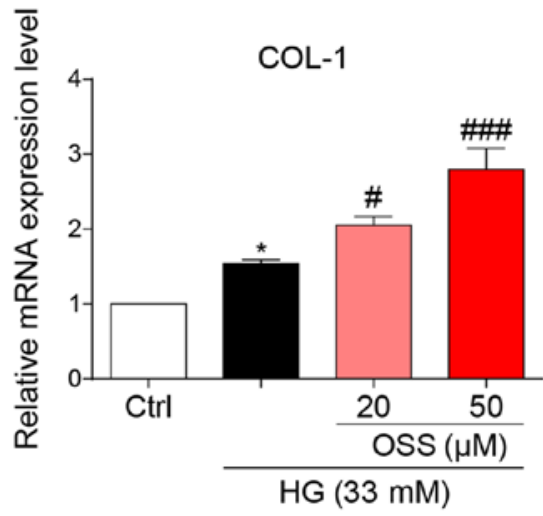

$\mathrm{D}$

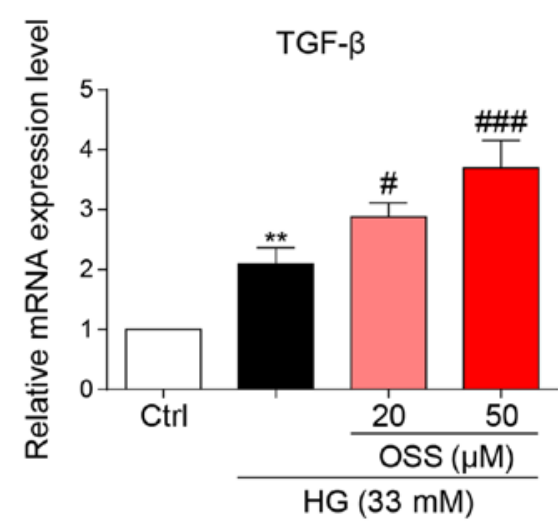

$\mathrm{F}$

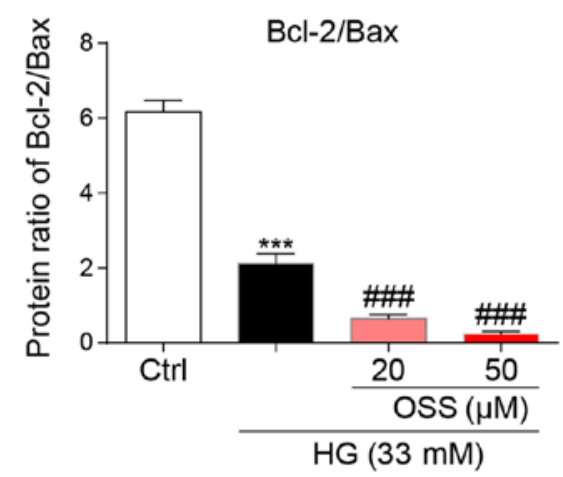

$\mathrm{H}$

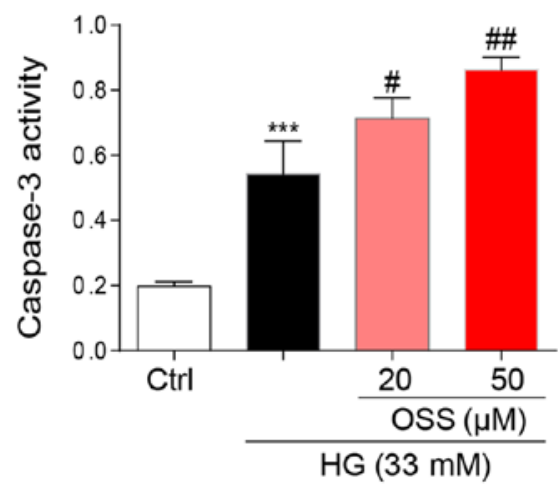

Figure 3. OSS-128167 aggravates HG-induced fibrosis and apoptosis of H9c2 cells. (A) Western blot analysis of COL-1 and TGF- $\beta$ in H9c2 cells. H9c2 cells underwent $1 \mathrm{~h}$ of pre-incubation with a recommended dose of OSS-128167, followed by $36 \mathrm{~h}$ of $33 \mathrm{mM}$ HG treatment. Proteins underwent immunoblotting, and GAPDH was used as a loading control. (B-D) H9c2 cells underwent $1 \mathrm{~h}$ of pre-incubation with a recommended dose of OSS-128167, followed by $12 \mathrm{~h}$ of $33 \mathrm{mM} \mathrm{HG}$ incubation. Reverse transcription-quantitative PCR was performed to determine the mRNA expression levels of COL- 1 , CTGF and TGF- $\beta$. (E and F) H9c2 cells underwent $1 \mathrm{~h}$ of pre-incubation with a recommended dose of OSS-128167, followed by $36 \mathrm{~h}$ of $33 \mathrm{mM}$ HG treatment. Western blot analysis of Bcl-2 and Bax in H9c2 cells. (G) Western blot analysis of cle-PARP in H9c2 cells. GAPDH was used as the loading control. (H) H9c2 cells underwent $1 \mathrm{~h}$ of pre-incubation with a recommended dose of OSS-128167, followed by $12 \mathrm{~h}$ of $33 \mathrm{mM}$ HG incubation. Caspase 3 activity assay kit was used to determine caspase 3 activity in $\mathrm{H} 9 \mathrm{c} 2$ cells. Data from three independent experiments are presented. ${ }^{*} \mathrm{P}<0.05,{ }^{* *} \mathrm{P}<0.01,{ }^{* * *} \mathrm{P}<0.001$ vs. Ctrl; ${ }^{*} \mathrm{P}<0.05$, ${ }^{\# \#} \mathrm{P}<0.01,{ }^{\# \# \#} \mathrm{P}<0.001$ vs. HG. HG, high glucose; COL-1, collagen 1 ; TGF- $\beta$, transforming growth factor- $\beta$; CTGF, connective tissue growth factor; Bcl-2, B-cell lymphoma 2; Bax, Bcl-2-associated X; cle-PARP, cleaved-poly ADP-ribose polymerase; Ctrl, control. 
A

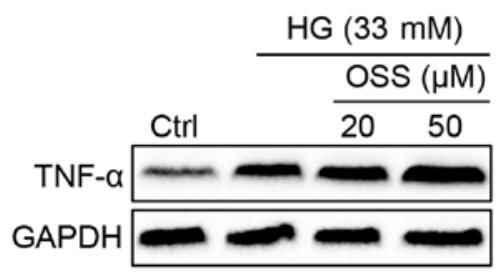

B
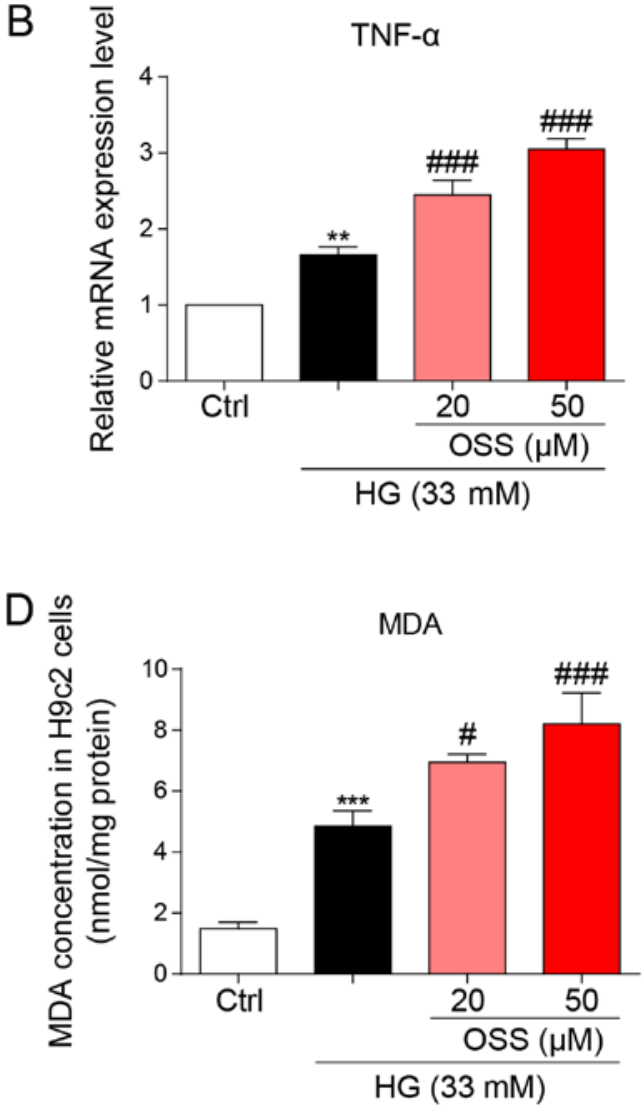

Figure 4. OSS-128167 aggravates HG-induced inflammation and oxidative stress in H9c2 cells. (A) H9c2 cells underwent 1 h of pretreatment with a recommended dose of OSS-128167, followed by 24 h of $33 \mathrm{mM} \mathrm{HG}$ treatment. Western blot analysis of TNF- $\alpha$ in H9c2 cells. GAPDH was used as a loading control. (B) H9c2 cells underwent 1 h of pretreatment with a recommended dose of OSS-128167, followed by 8 h of 33 mM HG incubation. Reverse transcription-quantitative PCR assay was used to detect the mRNA expression levels of TNF- $\alpha$. (C) H9c2 cells underwent $1 \mathrm{~h}$ of pretreatment with a recommended dose of OSS-128167, followed by $24 \mathrm{~h}$ of $33 \mathrm{mM} \mathrm{HG}$ treatment. Western blot analysis of 3-NT in H9c2 cells. GAPDH was used as a loading control. (D) Cells underwent $1 \mathrm{~h}$ of pre-treatment with a recommended dose of OSS-128167, followed by $4 \mathrm{~h}$ of HG (33 mM) treatment. MDA levels in H9C2 cells were detected. Data from three independent experiments are shown. ${ }^{* *} \mathrm{P}<0.01,{ }^{* * *} \mathrm{P}<0.001$ vs. Ctrl; ${ }^{\#} \mathrm{P}<0.05$, ${ }^{\# \#} \mathrm{P}<0.001$ vs. HG. HG, high glucose; TNF- $\alpha$, tumor necrosis factor- $\alpha$; 3-NT, 3-N-terminal; MDA, malondialdehyde; Ctrl, control.

activity was increased in the OSS-128167 group compared with that in the HG group (Fig. 3H). These results revealed that OSS-128167 could significantly increase myocardial fibrosis and the expression of apoptotic proteins induced by hyperglycemia in vivo.

OSS-128167 aggravates HG-induced H9c2 cell inflammation and oxidative stress. The protein and mRNA expression levels of the inflammatory cytokine, TNF- $\alpha$ were measured to evaluate the pro-inflammatory biological function of OSS-128167. OSS-128167 was revealed to significantly aggravate HG-induced increases in TNF- $\alpha$ expression levels (Fig. 4A and B). These findings were in accordance with the results of the in vivo studies and suggested the negative impact of OSS-128167 on cardiomyocytes through the aggravation of inflammation. Oxidative stress also serves as an important pathological mechanism of DCM. HG-stimulated $\mathrm{H} 9 \mathrm{c} 2$ cells underwent immunoblotting (Fig. 4C), and the expression levels of 3-NT, a biomarker of the nitrogen free radical species, exhibited markedly increased accumulation in H9c2 cells treated with HG which was more prominent in the OSS-128167-treated groups. Furthermore, MDA levels were measured in $\mathrm{H} 9 \mathrm{c} 2$ cells. Polyunsaturated fatty acids produce MDA via lipid peroxidation; in the present study, OSS-128167 aggravated the HG-induced increase in MDA (Fig. 4D). These results demonstrated that OSS-128167 aggravated HG-induced cell inflammatory responses and oxidative stress in $\mathrm{H} 9 \mathrm{c} 2$ cells.

\section{Discussion}

During the past few decades, the incidence and prevalence of diabetes mellitus have increased worldwide; this is primarily due to the increased incidence of diabetic complications, such as DCM (7). Accordingly, developing additional treatments and novel prevention strategies for DCM is urgently required. The present study revealed that the novel use of a SIRT6 inhibitor, OSS-128167, exacerbated cardiac structural alterations in diabetic mice. Moreover, the present study revealed that the cardio-pernicious effect of OSS-128167 also led to aggravated inflammation and oxidative stress. These findings were verified in vitro using $\mathrm{HG}$-treated $\mathrm{H} 9 \mathrm{c} 2$ cells.

Chronic and persistent inflammation, and oxidative stress markedly affect the pathophysiology of cardiovascular disorders induced by hyperglycemia. Persistent inflammatory factors and ROS caused by hyperglycemia have been shown to damage normal cellular functions and structure, causing apoptosis of cardiomyocytes $(25,26)$. Previous studies have demonstrated the benefits of implementing anti-inflammatory strategies for cardiac 
health $(27,28)$. In a previous study, overexpression of TNF- $\alpha$ exacerbated myocardial apoptosis by causing desmin cleavage and modification, eventually leading to heart failure (29). Additionally, the blockage of TNF- $\alpha$ was found to significantly alleviate the inflammatory response and myocardial fibrosis in the myocardial intima of patients with DCM (30). In the present study, OSS-128167 was observed to increase the expression levels of TNF- $\alpha$ caused by $\mathrm{HG}$ or diabetes in vitro and in vivo.

Another mechanism of oxidative stress-induced myocardial damage also significantly affects the pathophysiological mechanism of DCM. Oxidative stress has been shown to facilitate myocardial fibrosis in response to HG (31). According to previous studies, factors such as hyperglycemia, hyperlipidemia, increased free fatty acid levels and accumulated advanced glycosylation end products can promote the production of ROS and reactive nitrogen species in the diabetic myocardium $(32,33)$. In the present study, OSS-128167 was revealed to exacerbate oxidative stress in $\mathrm{HG}$-induced cardiomyocytes, with similar results being observed in the myocardium of type 1 diabetic mice.

Apoptosis and fibrosis of heart tissue are mediated by inflammatory responses and oxidative stress. Therefore, experiments were conducted to evaluate the biological effects of OSS-128167 on hyperglycemia/HG-mediated myocardial damage in vivo and in vitro. Hyperglycemia and $\mathrm{HG}$ induced the expression of pro-fibrotic markers (COL-1, TGF- $\beta$ and CTGF) and OSS-128167 significantly increased the expression of these fibrotic biomarkers. In addition, in STZ-induced diabetic mice, the number of apoptotic myocardial cells detected by TUNEL staining was markedly increased in the OSS-128167 treatment group compared with in the STZ-DM1 group. Subsequently, in $\mathrm{H} 9 \mathrm{c} 2$ cells under HG challenge treatment with OSS-128167 reduced the expression levels of the anti-apoptotic protein Bcl-2 expression, whereas it increased the expression levels of pro-apoptotic proteins Bax and cle-PARP. These results indicated that inhibiting the expression of SIRT6 could exacerbate hyperglycemia-induced myocardial damage by increasing the fibrosis and apoptosis of myocardial cells.

The present study revealed that $\mathrm{HG}$ induced cardiac inflammation and oxidative stress, which may lead to the progression of DCM. In addition, the present study provided a novel understanding of the regulatory role of SIRT6 in cardiac injury caused by HG. Notably, OSS-128167 was found to facilitate inflammation, oxidative stress, fibrosis and apoptosis both in vitro and in vivo. Although previous studies aimed to find new therapies to prevent or treat DCM, drugs or therapeutic targets that can completely reverse the process of DCM have not yet been discovered. These experiments largely demonstrated that SIRT6 may serve as a novel therapeutic target for DCM. However, the present study also has some limitations. Notably, the function of SIRT6 in DCM was not directly assessed; a specific inhibitor of SIRT6 was used instead. This lacks strength in explaining the role that SIRT6 has in DCM. Future studies should investigate additional intricate mechanisms of SIRT6 to determine if it may be used to treat diabetes and its complications.

\section{Acknowledgements}

Not applicable.

\section{Funding}

No funding was received.

\section{Availability of data and materials}

The datasets used and/or analyzed during the current study are available from the corresponding author on reasonable request.

\section{Authors' contributions}

LZ was the principal investigator, designed the study, supervised experiments and wrote the manuscript. YH, JZ, DX, YP and YJ performed experiments. YH, JZ and DX analyzed the data. All authors read and approved the final manuscript, and agree to be accountable for all aspects of the research in ensuring that the accuracy or integrity of any part of the work are appropriately investigated and resolved. $\mathrm{LZ}$ and $\mathrm{YH}$ confirm the authenticity of all the raw data.

\section{Ethics approval and consent to participate}

The animal raising and handling procedures were performed in accordance with the Guide for the Care and Use of Laboratory Animals. The present study was approved by the Wenzhou Medical University Animal Policy and Welfare Committee.

\section{Patient consent for publication}

Not applicable.

\section{Competing interests}

The authors declare that they have no competing interests.

\section{References}

1. Meagher P, Adam M, Civitarese R, Bugyei-Twum A and Connelly KA: Heart failure with preserved ejection fraction in diabetes: Mechanisms and management. Can J Cardiol 34: 632-643, 2018.

2. Whiting DR, Guariguata L, Weil C and Shaw J: IDF diabetes atlas: Global estimates of the prevalence of diabetes for 2011 and 2030. Diabetes Res Clin Pract 94: 311-321, 2011.

3. Al-Quwaidhi AJ, Pearce MS, Sobngwi E, Critchley JA and O'Flaherty M: Comparison of type 2 diabetes prevalence estimates in Saudi Arabia from a validated Markov model against the International Diabetes Federation and other modelling studies. Diabetes Res Clin Pract 103: 496-503, 2014.

4. Devereux RB, Roman MJ, Paranicas M, O'Grady MJ, Lee ET, Welty TK, Fabsitz RR, Robbins D, Rhoades ER and Howard BV: Impact of diabetes on cardiac structure and function: The strong heart study. Circulation 101: 2271-2276, 2000.

5. Varma U, Koutsifeli P, Benson VL, Mellor KM and Delbridge LMD: Molecular mechanisms of cardiac pathology in diabetes-Experimental insights. Biochim Biophys Acta Mol Basis Dis 1864: 1949-1959, 2018.

6. Dillmann WH: Diabetic cardiomyopathy. Circ Res 124: 1160-1162, 2019.

7. Levelt E, Gulsin G, Neubauer $S$ and McCann GP: MECHANISMS IN ENDOCRINOLOGY: Diabetic cardiomyopathy: Pathophysiology and potential metabolic interventions state of the art review. Eur J Endocrinol 178: R127-R139, 2018.

8. Tan Y, Zhang Z, Zheng C, Wintergerst KA, Keller BB and Cai L: Mechanisms of diabetic cardiomyopathy and potential therapeutic strategies: Preclinical and clinical evidence. Nat Rev Cardiol 17: 585-607, 2020. 
9. Jia G, Hill MA and Sowers JR: Diabetic cardiomyopathy: An update of mechanisms contributing to this clinical entity. Circ Res 122: 624-638, 2018.

10. Yang F, Qin Y, Wang Y, Li A, Lv J, Sun X, Che H, Han T, Meng S, Bai Y and Wang L: LncRNA KCNQ1OT1 mediates pyroptosis in diabetic cardiomyopathy. Cell Physiol Biochem 50: 1230-1244, 2018.

11. Feng W, Lei T, Wang Y, Feng R, Yuan J, Shen X, Wu Y, Gao J, Ding W and Lu Z: GCN2 deficiency ameliorates cardiac dysfunction in diabetic mice by reducing lipotoxicity and oxidative stress. Free Radic Biol Med 130: 128-139, 2019.

12. YuLM,Dong X, Xue XD, Xu S,Zhang X, Xu YL, WangZS, Wang Y, Gao H, Liang YX, et al: Melatonin attenuates diabetic cardiomyopathy and reduces myocardial vulnerability to ischemia-reperfusion injury by improving mitochondrial quality control: Role of SIRT6. J Pineal Res 70: e12698, 2021.

13. Jubaidi FF, Zainalabidin S, Mariappan V and Budin SB: Mitochondrial dysfunction in diabetic cardiomyopathy: The possible therapeutic roles of phenolic acids. Int J Mol Sci 21: $6043,2020$.

14. Finkel T, Deng CX and Mostoslavsky R: Recent progress in the biology and physiology of sirtuins. Nature 460: 587-591, 2009.

15. Michishita E, McCord RA, Berber E, Kioi M, Padilla-Nash H, Damian M, Cheung P, Kusumoto R, Kawahara TL, Barrett JC, et al: SIRT6 is a histone H3 lysine 9 deacetylase that modulates telomeric chromatin. Nature 452: 492-496, 2008.

16. Yang B, Zwaans BM, Eckersdorff $M$ and Lombard DB: The sirtuin SIRT6 deacetylates H3 K56Ac in vivo to promote genomic stability. Cell Cycle 8: 2662-2663, 2009.

17. Tennen RI and Chua KF: Chromatin regulation and genome maintenance by mammalian SIRT6. Trends Biochem Sci 36: 39-46, 2011.

18. Kugel S and Mostoslavsky R: Chromatin and beyond: The multitasking roles for SIRT6. Trends Biochem Sci 39: 72-81, 2014

19. Khan RI, Nirzhor SSR and Akter R: A review of the recent advances made with SIRT6 and its implications on aging related processes, major human diseases, and possible therapeutic targets. Biomolecules 8: 44, 2018.

20. Singh CK, Chhabra G, Ndiaye MA, Garcia-Peterson LM, Mack NJ and Ahmad N: The role of sirtuins in antioxidant and redox signaling. Antioxid Redox Signal 28: 643-661, 2018.

21. Arsiwala T, Pahla J, van Tits LJ, Bisceglie L, Gaul DS, Costantino S, Miranda MX, Nussbaum K, Stivala S, Blyszczuk P, et al: Sirt6 deletion in bone marrow-derived cells increases atherosclerosis-Central role of macrophage scavenger receptor 1. J Mol Cell Cardiol 139: 24-32, 2020.

22. Jin Z, Xiao Y, Yao F, Wang B, Zheng Z, Gao H, Lv X, Chen L, He Y Wang W and Lin R: SIRT6 inhibits cholesterol crystal-induced vascular endothelial dysfunction via Nrf2 activation. Exp Cell Res 387: 111744, 2020.
23. Wang L, Han J, Shan P, You S, Chen X, Jin Y, Wang J, Huang W, Wang Y and Liang G: MD2 blockage protects obesity-induced vascular remodeling via activating AMPK/Nrf2. Obesity 25: 1532-1539, 2017

24. Livak KJ and Schmittgen TD: Analysis of relative gene expression data using real-time quantitative PCR and the 2(-Delta Delta $\mathrm{C}(\mathrm{T})$ ) method. Methods 25: 402-408, 2001

25. Fang ZY, Prins JB and Marwick TH: Diabetic cardiomyopathy: Evidence, mechanisms, and therapeutic implications. Endocr Rev 25: 543-567, 2004.

26. Lee TW, Kao YH, Chen YJ, Chao TF and Lee TI: Therapeutic potential of vitamin D in AGE/RAGE-related cardiovascular diseases. Cell Mol Life Sci 76: 4103-4115, 2019.

27. Kolpakov MA, Sikder K, Sarkar A, Chaki S, Shukla SK, Guo X, Qi Z, Barbery C, Sabri A and Rafiq K: Inflammatory serine proteases play a critical role in the early pathogenesis of diabetic cardiomyopathy. Cell Physiol Biochem 53: 982-998, 2019.

28. Zhang X, Zhang Z, Yang Y, Suo Y, Liu R, Qiu J, Zhao Y, Jiang N, Liu C, Tse G, et al: Alogliptin prevents diastolic dysfunction and preserves left ventricular mitochondrial function in diabetic rabbits. Cardiovasc Diabetol 17: 160, 2018.

29. Panagopoulou P, Davos CH, Milner DJ, Varela E, Cameron J, Mann DL and Capetanaki Y: Desmin mediates TNF-alpha-induced aggregate formation and intercalated disk reorganization in heart failure. J Cell Biol 181: 761-775, 2008.

30. Westermann D, Van Linthout S, Dhayat S, Dhayat N, Schmidt A, Noutsias M, Song XY, Spillmann F, Riad A, Schultheiss HP and Tschöpe C: Tumor necrosis factor-alpha antagonism protects from myocardial inflammation and fibrosis in experimental diabetic cardiomyopathy. Basic Res Cardiol 102: 500-507, 2007.

31. Palomer X,Pizarro-Delgado J and Vazquez-Carrera M: Emerging actors in diabetic cardiomyopathy: Heartbreaker biomarkers or therapeutic targets? Trends Pharmacol Sci 39: 452-467, 2018

32. Sun HJ, Xiong SP, Wu ZY, Cao L, Zhu MY, Moore PK and Bian JS: Induction of caveolin-3/eNOS complex by nitroxyl (HNO) ameliorates diabetic cardiomyopathy. Redox Biol 32: 101493, 2020.

33. Barbeau PA, Holloway TM, Whitfield J, Baechler BL, Quadrilatero J, van Loon LJC, Chabowski A and Holloway GP: $\alpha$-Linolenic acid and exercise training independently, and additively, decrease blood pressure and prevent diastolic dysfunction in obese Zucker rats. J Physiol 595: 4351-4364, 2017.

This work is licensed under a Creative Commons Attribution-NonCommercial-NoDerivatives 4.0 International (CC BY-NC-ND 4.0) License. 